\title{
New mechanism of runaway electron diffusion due to microturbulence in tokamaks
}

S. S. Abdullaev, K. H. Finken, and M. Forster

Citation: Phys. Plasmas 19, 072502 (2012); doi: 10.1063/1.4736718

View online: http://dx.doi.org/10.1063/1.4736718

View Table of Contents: http://pop.aip.org/resource/1/PHPAEN/v19/i7

Published by the American Institute of Physics.

\section{Additional information on Phys. Plasmas}

Journal Homepage: http://pop.aip.org/

Journal Information: http://pop.aip.org/about/about_the_journal

Top downloads: http://pop.aip.org/features/most_downloaded

Information for Authors: http://pop.aip.org/authors

\section{ADVERTISEMENT}

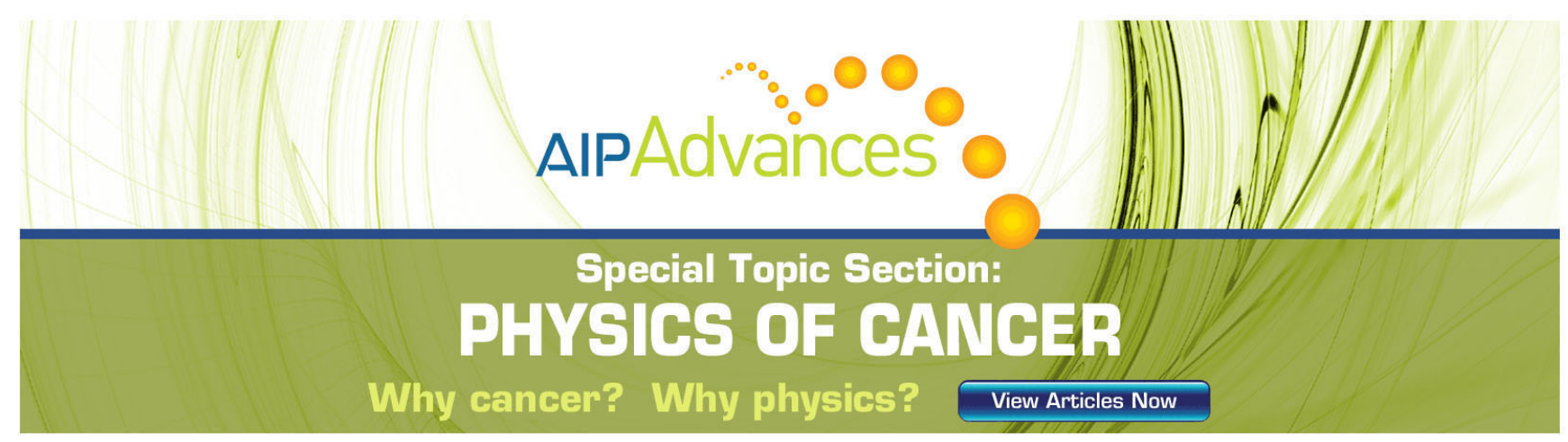




\title{
New mechanism of runaway electron diffusion due to microturbulence in tokamaks
}

\author{
S. S. Abdullaev, ${ }^{1}$ K. H. Finken, ${ }^{1,2}$ and M. Forster ${ }^{2}$ \\ ${ }^{1}$ Institute for Energy and Climate Research-Plasma Physics, Forschungszentrum Jülich GmbH, Association \\ EURATOM-FZJ, Partner in the Trilateral Euregio Cluster, D-52425 Jülich, Germany \\ ${ }^{2}$ Institut für Laser und Plasmaphysik, Heinrich-Heine-Universität Düsseldorf, Universitätsstr.1, \\ 40225 Düsseldorf, Germany
}

(Received 28 March 2012; accepted 29 May 2012; published online 11 July 2012)

\begin{abstract}
Chaotic transport of runaway electrons in a toroidal system in the presence of a weak small-scale magnetic turbulent field with a wide mode spectrum is studied. Using a fast running mapping, the radial profiles of turbulent diffusion coefficients are calculated. It is found that at large Kubo numbers the chaotic transport of the electrons is described by a fractal-like radial dependence of the diffusion coefficients with reduced or zero values near low-order rational drift surfaces which form transport barriers. The latter can be one of the main reasons of the improved confinement of runaway electrons in tokamaks. One can expect that this effect may lead to the formation of the nested beams of runaway electrons. [http://dx.doi.org/10.1063/1.4736718]
\end{abstract}

\section{INTRODUCTION}

The transport of runaway electrons in magnetically confined plasmas has been a subject of many studies over three decades (see, e.g., Refs. 1-8). Understanding this problem is of crucial importance in modern fusion devices. Runaway electrons, which are generated during the start-up or during disruptions of plasma discharges, have to be mitigated as they may cause substantial damage of the plasma facing wall components of fusion devices (see, Refs. 9-14 and references therein).

A magnetic turbulence is considered as a main cause of the radial transport of runaway electrons, which may reach energies from a few $\mathrm{MeV}$ up to several tens $\mathrm{MeV}$. Due to their high velocity, they are hardly sensitive to electrostatic turbulence like the thermal particles. This fact has been used to study the magnetic turbulence in tokamaks by the measurement of runaway electron diffusion. ${ }^{15,16}$

Theoretical studies of the runaway electron transport due to magnetic turbulence have been based on the assumption that it is mainly caused by the stochastic diffusion in a braided magnetic field, i.e., due to a magnetic diffusion. ${ }^{17}$ Thereby, the diffusion coefficients of runaway electrons, $D_{r}$, can be expressed via the magnetic field line diffusion coefficient, $D_{M}$ (Refs. 2, 3, and 18), that is $D_{r} \simeq \Upsilon v_{\|} D_{M}$, where $v_{\|} \lesssim c$ is the parallel velocity of the runaway electrons which is close to the speed of light $c$. The factor $\Upsilon$, known as a shielding factor, describes the deviation of the runaway electron diffusion from the thermal electron diffusion due to the displacement of the runaway electron orbits from the magnetic surfaces (orbitaveraging) and their large gyro-radii, i.e., gyro-averaging. The latter means that the runaway electrons do not experience the full strength of the magnetic turbulence which is located at the resonant surfaces. ${ }^{3-6,18}$ In Ref. 8 , the validity of the orbitaveraging due to the so-called orbit decorrelation effect and its role in the possible decrease of the runaway diffusion has been discussed.
The general asymptotical formula for the diffusion coefficients of runaway electrons which takes into account these facts has been derived in Ref. 19. According to this approach, the decrease of the diffusive transport with increasing electron energies occurs at small radii due to the effect of the gyroaveraging of a turbulent field and at large radii due to the orbit averaging effect. The latter is believed to be responsible for the improved confinement of runaway electrons with increasing energies.

In spite of these theoretical studies, the basic mechanisms of the runaway electron transport in a microturbulent magnetic field are not well understood. One of the critical issues is the validity of the above formula for the diffusion coefficients $D_{r}$ for the runaway electrons. The knowledge of $D_{r}$ is necessary to study the dynamics of runaway electron populations during tokamak discharges and to estimate the level of radial magnetic field fluctuations (see, e.g., Refs. 7 and 20-23).

In the present work, we tackle this problem by using a more general, $a b$ inito approach. The microturbulence in tokamaks is presented as fluctuating small-scale magnetic field perturbations with a wide range of modes and a finite correlation time. The corresponding equations of motion of the runaway electrons are directly integrated. This approach allows one to explore the dependence of the diffusive transport of the runaway electrons not only on the level of the turbulence field but also on its spectrum and the correlation time $\tau_{c}$. The effect of the latter can be presented by the Kubo number $K=\tau_{c} / T_{o r b}$, i.e., the ratio of the correlation time $\tau_{c}$ to the characteristic period $T_{o r b}$ of a particle orbit (see, e.g., Ref. 24). Particularly, it would also allow one to explore the validity of the quasilinear approximation and the condition at which stochastic or fluctuating mechanisms of the transport are dominant.

However, such an approach encounters computational difficulties. Since the turbulent field may contain a large number of modes which may extend up to several hundreds, the corresponding equations of motion have a large number 
of high-frequency oscillating terms. The standard methods to integrate such equations require huge computational times in order to get a sufficient accuracy. To overcome these technical obstacles, we employ the canonical mapping procedure with a mapping step equal to one full poloidal turn. Recently, this method has been applied in Ref. 25 to study the chaotic transport in Hamiltonian systems perturbed by a turbulent wave-field.

Another problem is related to the presentation of microturbulent fields in tokamaks. The properties of microturbulent magnetic fields, such as spectra, a correlation time, etc., are not well known. Since we are interested in the general features of the runaway electron transport, the specific values of the turbulent field characteristics are not necessary. We present the turbulent magnetic field as a sum of the large number $(m, n)$-modes localized near the corresponding resonant magnetic surfaces similar to the ones in Refs. 3-5 and 18.

We mainly study radial profiles of the diffusion coefficients of runaway electrons. Such a study allows one to quantitatively explore one of the mechanisms of the radial transport of runaway electrons, particularly, the role of low order rational surfaces in the particle transport. It will be shown that the chaotic transport of runaway electrons across drift surfaces may be drastically reduced near the low-order rational drift surfaces thereby forming transport barriers. We will study the condition at which such transport barriers are formed and their dependence on the mode spectra.

One should note that a possible role of the low-order rational magnetic surfaces on the formation of the electron and ion transport barriers has been discussed in a number of works since the early 1990s (see, e.g., Refs. 26-39 and references therein). It has been postulated that the appearance of the transport barriers is due to the presence of gaps in the density of the rational magnetic surfaces near the rational magnetic surfaces with low $m$ and $n$ numbers. A recent numerical study of the test particle transport in the ad hoc chaotic Hamiltonian systems shows that one of the conditions for the formation of such transport barriers is a large Kubo number of particles. ${ }^{25}$ This condition is well satisfied for the runaway electrons.

The paper consists of seven sections and an Appendix. The basic Hamiltonian equations for the relativistic guidingcenter motion are given in Sec. II. The model of a microturbulent magnetic field used for the calculations is described in Sec. III. The gyro-averaged Hamiltonian equation in the presence of a turbulent magnetic field is presented and analyzed in Sec. IV. The fast symplectic mapping used to integrate the Hamiltonian equations is presented in Sec. V. The radial transport of runaway electrons in the turbulent field is studied in Sec. VI using the quasilinear approximation and the direct numerical calculations. In the conclusive section VII, the obtained results are summarized and analyzed. In Appendix, the density of rational drift surfaces is calculated and their properties are analyzed.

\section{HAMILTONIAN EQUATIONS}

We use the version of the relativistic Hamiltonian equations for a guiding center motion in a toroidal system given in Refs. 40-42. These equations are obtained by the canonical change of gyrating particle variables to the guidingcenter ones. Since the runaway electrons are passing particles, we will use a simplified version of the Hamiltonian drift equations which are reformulated by introducing the toroidal angle $\varphi$ as the independent, time-like variable and the corresponding canonical momentum $p_{\varphi}$ as a new Hamiltonian $H=-p_{\varphi}$. The Hamiltonian equations for the canonical guiding-center variables $\left(q_{1}, q_{2}, p_{1}, p_{2}\right)=\left(z, T, p_{z},-E\right)$ are given (see Refs. 40 and 42)

$$
\frac{d q_{i}}{d \varphi}=\frac{\partial H}{\partial p_{i}}, \quad \frac{d p_{i}}{d \varphi}=-\frac{\partial H}{\partial q_{i}}, \quad(i=1,2) .
$$

The simplified form of the Hamiltonian function $H=H$ $\left(z, T, p_{z},-E, \varphi\right)$ is given by

$$
H=-f_{\varphi}-\sigma\left(R_{c} / R_{0}\right) \sqrt{\varepsilon_{0}\left(\gamma^{2}-1\right)-2 \omega_{R} I_{R}} .
$$

In (1), $z$ and $T$ are the normalized vertical coordinate $Z$ and the time $t: z=Z / R_{0}$ and $T=\omega_{c} t, p_{z}$ is a momentum conjugated to $z$. Here, $\omega_{c}=e B_{0} / m c$ is a gyrofrequency and $c$ is the speed of light in vacuum. The relativistic factor is $\gamma=E / \varepsilon_{0}$, where $\varepsilon_{0}=\left(c / \omega_{c} R_{0}\right)^{2}$ is a normalized energy of the particle at rest, $E$ is the particle energy. The quantity $f_{\varphi}(R, Z, \varphi, t)=R A_{\varphi} / B_{0} R_{0}^{2}$ is the normalized vector potential, $I_{R}$ is the action variable conjugated to the radial gyro-phase $\vartheta_{R}=\omega_{R} T+\vartheta_{R 0}$ describing the fast gyro-oscillations along the radial coordinate $R$, where the radial gyrofrequency $\omega_{R}$ is given by $\omega_{R}=e^{-p_{z}}$. The quantity $R_{c}=R_{0} e^{p_{z}}$ is the radial coordinate of a guiding-center. The parameter $\sigma=1$ for the runaway electrons in the TEXTOR tokamak (see Ref. 19).

The ratio $\lambda=T_{R} / T_{K}$ of the gyromotion energy, $T_{R}=\sqrt{\varepsilon_{0}^{2}+2 \omega_{R} I_{R}}-\varepsilon_{0}$, to the full kinetic energy of a particle, $T_{K}=\varepsilon_{0}(\gamma-1)$, is considered as the initial parameter of motion. From the experimental measurements, it follows that the parameter $\lambda$ may reach a value of 0.1 (Refs. 43 and 44). Furthermore, we will assume that $\lambda \leq 0.1$.

The particle coordinates $(R, Z, \varphi)$ are related to the guiding center variables $\left(z, p_{z}\right)$,

$$
R=R_{c}+\rho \sin \vartheta_{R}, \quad Z=R_{0} z+\rho \cos \vartheta_{R},
$$

where $\rho=R_{0} e^{p_{z} / 2} \sqrt{2 I_{R}}$ is the radial gyroradius.

In the equilibrium plasma, the Hamiltonian $H_{0}=H$ $\left(z, p_{z}, E\right)$ is independent of the toroidal angle $\varphi$ and the time $t$. The particle guiding center orbits lie on the toroidal drift surfaces given by $H_{0}=$ const. For the description of such a motion, it is convenient to introduce the action-angle variables $(J, \vartheta)$ associated with the variables $\left(z, p_{z}\right)$. Then, $H_{0}=H$ $(J, E)$ and the position of a guiding center on the drift surface at the time instant $t$ is determined by linear functions of $\varphi$,

$$
\vartheta=\varphi / q_{p}(J, E)+\vartheta_{p 0}, \quad t=\varphi \nu_{t}(J, E) / 2 \pi+t_{0},
$$

where $q_{p}(J, E)=1 /\left(\partial H_{0} / \partial J\right)$ is an effective safety factor. Similar to the safety factor $q_{M}(\psi)$ of the magnetic field it is equal to the number of toroidal turns made by a particle 


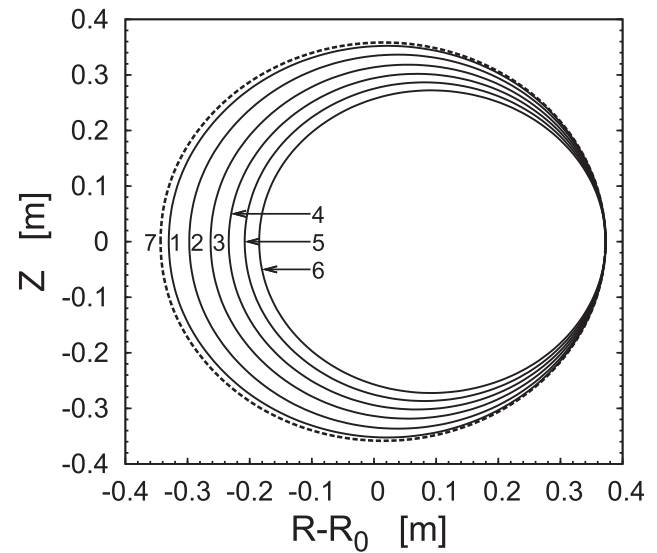

FIG. 1. Drift orbits of runaway electrons of different energies: Curve 1 corresponds to $E=1 \mathrm{MeV}$; curve 2, $E=5 \mathrm{MeV}$; curve 3, $E=10 \mathrm{MeV}$; curve 4, $E=15 \mathrm{MeV}$; curve $5, E=20 \mathrm{MeV}$; and curve $6, E=25 \mathrm{MeV}$. Dashed curve 7 corresponds to the field line magnetic surface, which has a common point with the particle drift orbits at the low field side. The parameter $\lambda=0.1$. The plasma parameters: the major radius $R_{0}=1.75 \mathrm{~m}$, the minor radius $a=0.46 \mathrm{~m}$, the toroidal field $B_{t}=2.2 \mathrm{~T}$, the plasma current $I_{p}=0.3 \mathrm{MA}$, the plasma $\beta_{\text {pol }}=0.3$.

guiding center during one poloidal turn. At the limit $E \rightarrow 0$, the action variable $J$ and $q_{p}(J, E)$ coincide with the normalized toroidal flux $\psi$ and $q_{M}(\psi)$, respectively. The quantity $\nu_{t}(J, E)=\partial H_{0} / \partial E$ is a transit time for a particle to make a full toroidal turn.

The deviation of the drift surfaces from the magnetic ones is illustrated in Fig. 1. It shows the poloidal sections of the drift surfaces corresponding to different electron energies ranging from $E=1 \mathrm{MeV}$ up to $E=25 \mathrm{MeV}$ (curves 1-6, respectively). They have common points at the section $\vartheta=0$. The dashed curve 7 corresponds to the magnetic flux surfaces touching the drift surfaces at $\vartheta=0$. The plasma parameters correspond to the typical TEXTOR discharges with the major radius $R_{0}=1.75 \mathrm{~m}$, the minor radius $a=0.46 \mathrm{~m}$, $B_{t}=2.2 \mathrm{~T}, I_{p}=0.3 \mathrm{MA}$, and $\beta_{p o l}=0.3$. As seen from Fig. 1 , due to the toroidal effects the drift surfaces of the runaway electrons do not coincide with the magnetic surfaces and the geometrical centers of the drift surfaces are shifted outward with respect to the one of the magnetic surfaces.

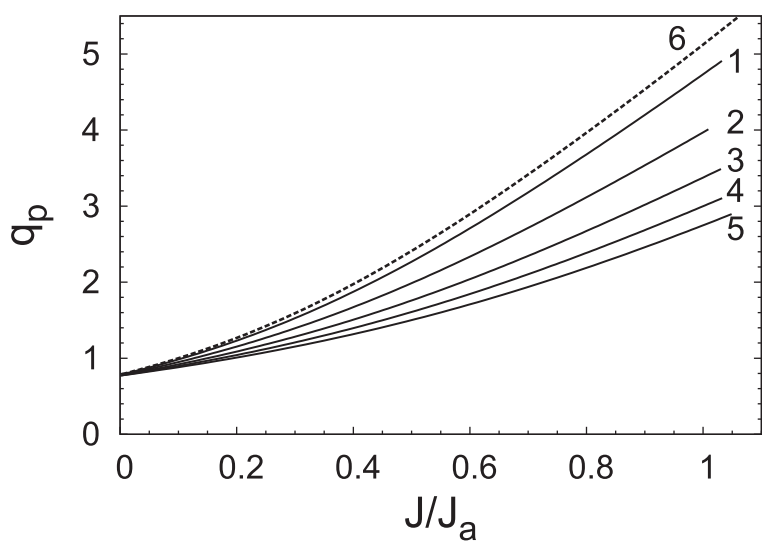

FIG. 2. Effective safety factors, $q_{p}(J, E)$ for runaway electrons: Curve 1 corresponds to $E=1 \mathrm{MeV}$, curve 2 to $E=5 \mathrm{MeV}$, curve 3 to $E=10 \mathrm{MeV}$, curve 4 to $E=15 \mathrm{MeV}$, curve 5 to $E=20 \mathrm{MeV}$, and curve 6 describes the safety factor of magnetic field.
Figure 2 shows the dependencies of the effective safety factors $q_{p}(J, E)$ of the runaway electrons on the normalized action variable $J / J_{a}$, where $J_{a}$ is the value of $J$ at the plasma edge $r=a$. In Fig. 2, the curves 1, 2, 3, 4, and 5, correspond to the electron energies $E=1,5,10,15$, and $20 \mathrm{MeV}$, respectively. Curve 6 corresponds to the safety factor $q_{M}(\psi)$ of the equilibrium magnetic field.

\section{TURBULENT MAGNETIC FIELD}

The nature of the magnetic fluctuations in tokamak plasmas, as well as electric field fluctuations, is not well known. Therefore, we consider the small-scale magnetic fluctuations by presenting them as a sum of a large number of $(m, n)$ modes radially localized near the corresponding resonant magnetic surfaces without specifying the nature of the magnetic turbulence. Similar presentations of the turbulent magnetic field have been used in Refs. 3-5 and 18.

\section{A. Presentation of a turbulent magnetic field}

Let $\left(\psi, \vartheta_{M}, \varphi\right)$ be magnetic field coordinates in which the field lines are straight. We present the toroidal component of the vector potential of the turbulent magnetic field as a sum of $(m, n)$-modes with random phases $\chi_{m n}$,

$$
\begin{aligned}
A_{\varphi}^{(1)}(R, Z, \varphi)= & \epsilon B_{0} \sum_{m n} r m^{-1} b_{m n}(\psi) \cos \left(m \vartheta_{M}\right) \\
& \times \cos \left(n \varphi+\chi_{m n}\right)
\end{aligned}
$$

where $\epsilon=\sigma_{A} /\left|A_{\varphi}^{(0)}(R, Z)\right|$ stands for the dimensionless amplitude of the turbulent magnetic field, i.e., it is determined by the ratio of the amplitude of the magnetic fluctuations, $\delta B=\nabla \times A_{\varphi}^{(1)}$ to the toroidal field strength, $B_{0}$, i.e., $\epsilon \sim \delta B / B_{0}$. The functions $b_{m n}(\psi)$ describe the radial $(\psi-)$ dependence of the $(m, n)$-mode. They are localized near the resonant magnetic surfaces $\psi_{m n}$ determined by the condition $q\left(\psi_{m n}\right)=m / n$.

The magnetic fluctuations contain a wide range of poloidal and toroidal modes $(m, n)$. In a toroidal system, one can assume that for the given toroidal mode $n$ the different poloidal modes $m$ are not independent but they are coupled due to a toroidicity. On the other hand, one can suppose that the modes belonging to the different toroidal modes $n$ are statistically independent. It allows one to assume that the random phases $\chi_{m n}(t)$ depend only on the toroidal mode number $n$, i.e., $\chi_{n}(t) \equiv \chi_{m n}(t)$. Furthermore, we will assume that the turbulent fluctuations are a stationary random process in time with the correlation time $\tau_{c}$.

One of the important parameters of the magnetic turbulence is its fluctuation level $\delta B$. For the latter, we consider the magnetic turbulence caused by microtearing modes. According to the theory by Drake et ll $^{45}$ the nonlinear saturation level of $\delta B$ driven by the electron temperature gradient is given by $\delta B / B_{0} \simeq \rho_{e} / L_{T_{e}}$, where $\rho_{e}$ is the thermal electron gyroradius, $L_{T_{e}}=T_{e} /\left(d T_{e} / d r\right)$ is the characteristic length of the variation of the electron temperature $T_{e}$. The recent gyrokinetic simulations ${ }^{46}$ show that the Drake formula quite well describes the level of magnetic fluctuations, and it can be used for rough estimations of $\delta B$. For the TEXTOR 
parameters, $T_{e} \approx 1 \mathrm{keV}, L_{T_{e}}=0.1 \mathrm{~m}$, and $B_{0}=2.2 \mathrm{~T}$, we have $\delta B / B_{0} \sim 10^{-4}$.

The transport of particles in a fluctuating magnetic field depends on the Kubo number,

$$
K=\frac{v_{\|} \tau_{c}}{l_{c}}=\frac{\tau_{c}}{T q_{p}}
$$

defined as the ratio of the correlation time $\tau_{c}$ to the characteristic transit time $T(J, E) q_{p}$, where $v_{\|}$is the parallel velocity of a particle and $l_{c}=2 \pi R_{0} q_{p}$ is the connection length (see, e.g., Ref. 24). This definition of the Kubo number $K$ is used in Refs. 47 and 48 in the problem of the test particle transport in the turbulent field. Such a definition of $K$ is, probably, more adequate in our problem of the runaway electron transport in a fluctuating magnetic field than the Kubo number defined in Ref. 49 for the transport of the magnetic field lines in a turbulent magnetic field.

The typical value of the correlation time of a turbulent field is of the order of $\tau_{c} \sim 10^{-5} \mathrm{~s}$. The radial profiles of the Kubo number $K=K(J, E)(6)$ are shown in Fig. 3 for different energies of the runaway electrons. It is assumed that the correlation time $\tau_{c}$ of the turbulent field is $10^{-5} \mathrm{~s}$ at all radial locations. As seen from Fig. 3, the Kubo number is of the order of $10^{2}$ for the runaway electrons of energies $E \gtrsim 1 \mathrm{MeV}$. Such electrons "feel" the magnetic turbulent field as a frozen one and as we will see later their radial transport is mainly caused by the interactions of resonances.

\section{B. A specific model of modes}

For our calculations, we should specify the model for the turbulent magnetic field. Suppose that the amplitude, $b_{m n}(\psi)$, of the $(m, n)$-mode is described by the following radially dependent function:

$$
b_{m n}(\psi)=A(\psi) c_{n} \exp \left[-(m-n q(\psi))^{2} / 4 w_{n}^{2}\right],
$$

where $A(\psi)$ describes the spatial radial dependence of the amplitude of the magnetic fluctuations and $c_{n}$ is its toroidal spectrum. The poloidal mode spectrum of a given toroidal mode $n$ is localized near the resonant magnetic surface $\psi_{m n}$, $q\left(\psi_{m n}\right)=m / n$. The quantities $w_{n}$ are the widths of the

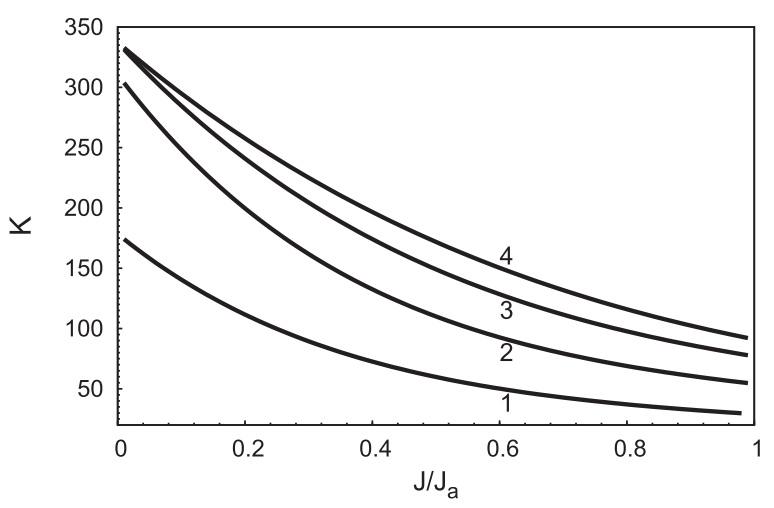

FIG. 3. Radial profiles of the Kubo number $K(J, E)(6)$ for runaway electrons with the different energies: Curve 1 corresponds to $E=0.1 \mathrm{MeV}$, curve 2 to $E=1 \mathrm{MeV}$, curve 3 to $E=10 \mathrm{MeV}$, curve 4 to $E=20 \mathrm{MeV}$. The other parameters are the same as in Fig. 2. The correlation time $\tau_{c}$ is taken equal $10^{-5} \mathrm{~s}$. poloidal mode spectra of a given toroidal mode $n$. For our model, we choose the following power-law distribution of the toroidal modes, $c_{n}=n^{-\alpha}$, with the constant parameter $\alpha$. For simplicity, we assume that the magnetic fluctuation level $A(\psi)$ is radially homogeneous, i.e., $A(\psi)=$ constant.

According to Eq. (5), the magnitude of the radial component of the turbulent magnetic field $B_{r}=r^{-1} \partial A_{\varphi} / \partial \theta$ can be reduced to

$$
\delta B=\sqrt{\left\langle B_{r}^{2}\right\rangle} \approx \frac{\epsilon B_{0}}{2}\left(\sum_{n=n_{\min }}^{n_{\max }} \sum_{m}\left|b_{m n}(\psi)\right|^{2}\right)^{1 / 2},
$$

where $\langle(\cdots)\rangle$ stands for an averaging over random phases $\chi_{n}$ and the poloidal angle $\vartheta_{M}$. Using Eq. (7) and assuming that $w_{n} \equiv w$ and $n_{\max } \gg n_{\min }$, one can obtain the following estimation: $\delta B \simeq \epsilon B_{0} C$, where $C=(\sqrt{2 \pi} w)^{1 / 2} / 2 n_{\min }^{\alpha-1 / 2}$. This estimation is close to the one calculated numerically according to Eq. (8). The latter is plotted in Fig. 4 for three different values of the exponent $\alpha$ with a fixed mode width $w$.

\section{PERTURBATION HAMILTONIAN}

Using the action-angle variables $(J, \vartheta)$ and the relation (3), the Hamiltonian equations (1) in the presence of nonaxisymmetric magnetic perturbations (5) can be reduced to (see, e.g., Refs. 19 and 41)

$$
\begin{aligned}
\frac{d Q_{i}}{d \varphi} & =\frac{\partial H}{\partial P_{i}}, \quad \frac{d P_{i}}{d \varphi}=-\frac{\partial H}{\partial Q_{i}}, \quad(i=1,2), \\
H & =H_{0}(J, E)+H_{1}(J, E, \vartheta, T, \varphi),
\end{aligned}
$$

where $\left(Q_{1}, Q_{2}, P_{1}, P_{2}\right)=(\vartheta, T, J, E)$. The perturbation Hamiltonian $H_{1}(J, E, \vartheta, T, \varphi)$ is obtained by averaging over the gyro-phase $\vartheta_{R}$.

Because of the periodicity of the system in the poloidal, $\vartheta$, and the toroidal, $\varphi$, directions the perturbation Hamiltonian $H_{1}$ can be expanded in a Fourier series in $\vartheta$ and $\varphi$,

$$
H_{1}=\sum_{m, n} H_{m n}(J, E) e^{i(m \vartheta-n \varphi)},
$$

where the Fourier coefficients $H_{m n}(J, E)=\left|H_{m n}(J, E)\right| e^{i \chi_{m n}(t)}$ are determined by the turbulent magnetic field.

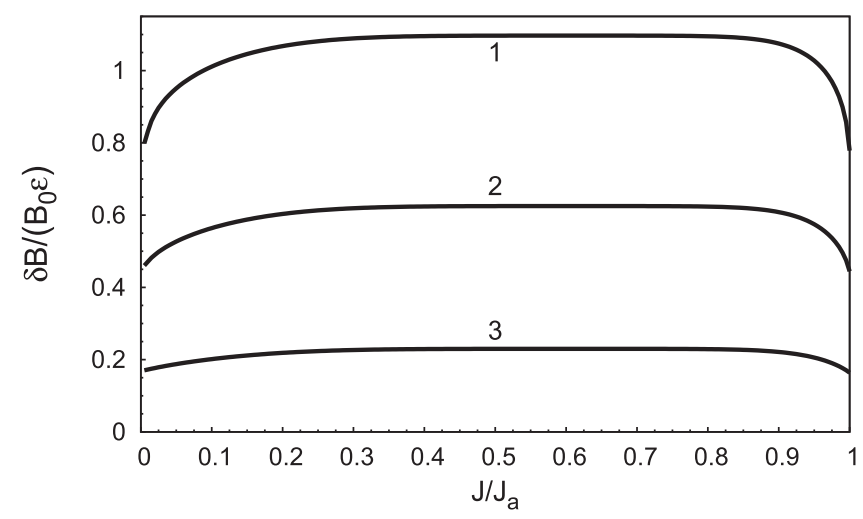

FIG. 4. Radial profiles of the magnitude of the turbulent magnetic field $\delta B$ (8) normalized to $\epsilon B_{0}$ for the three different values of the exponent $\alpha$ : Curve 1 corresponds to $\alpha=5 / 6$, curve 2 to $\alpha=1$, and curve 3 to $\alpha=4 / 3$. The width of the mode $w=6$, and $n_{\min }=10$. 
In general, the poloidal mode spectrum $H_{m n}$ for the given toroidal mode $n$ does not coincide with the corresponding spectrum of the turbulent field $b_{m n}$ but depends on the particle energy, its gyro-radius $\rho_{g}$, as well as on the direction of the particle motion due to the deviations of the drift orbits from the magnetic surfaces and gyro-averaging of the turbulent fluctuations. For large $m \gg 1$, the relation between $H_{m n}$ and $b_{m n}$ is described by the asymptotical formula of the type (see Ref. 19)

$$
H_{m n}\left(J, I_{R}, E\right) \approx e^{i \chi_{n}} \frac{C}{m \gamma_{1}} J_{0}\left(\frac{m \rho_{g}}{r \gamma_{1}}\right) b_{m^{\prime} n},
$$

where $m^{\prime} \approx m / \gamma_{1}, r$ is a minor radius, $J_{0}(z)$ is the Bessel function, and $C$ is a factor weakly depending on $J$. The parameter $\gamma_{1},\left(\gamma_{1} \neq 1\right)$, takes into account the drift of the guiding center orbit with respect to the magnetic surface. It is given by the first derivative of the poloidal angle $\vartheta_{M}$ of the magnetic field with respect to the corresponding angle $\vartheta$ of the guiding center taken on the low field side $(\vartheta=0)$ of the torus, i.e., $\gamma_{1}=d \vartheta_{M} / d \vartheta$ at $\vartheta=0$.

The typical radial dependencies of the parameter $\gamma_{1}$ at different values of the runaway electron energy are plotted in Fig. 5.

From the asymptotical form (11) of $H_{m n}$, it follows that for the given toroidal mode $n$ the poloidal mode $m$ of the perturbation spectra $H_{m n}$ is determined by the $m^{\prime} \approx m / \gamma_{1}$ poloidal mode of the turbulent field. This effect leads to the dependence of the turbulent transport on the particle energy. In Eq. (11), the term with the Bessel function $J_{o}(m \delta)$ takes into account the gyro-averaging effect of the turbulent field modes $m$, which effectively cuts off the contributions of the higher poloidal modes $m>m_{c}$ to the turbulent transport, where $m_{c}=\gamma_{1} r / \rho_{g}$ is the critical poloidal mode number. The corresponding toroidal mode number $n_{c}$ is equal to $n_{c}=m_{c} / q_{p}=\gamma_{1} r / q_{p} \rho_{g}$.

The typical radial dependencies of $n_{c}$ are shown in Fig. 6 for different energies of the runaway electrons.

\section{FAST SYMPLECTIC MAPPING}

Numerical simulations of the particle transport in a turbulent field with a large number of $(m, n)$-modes encounter

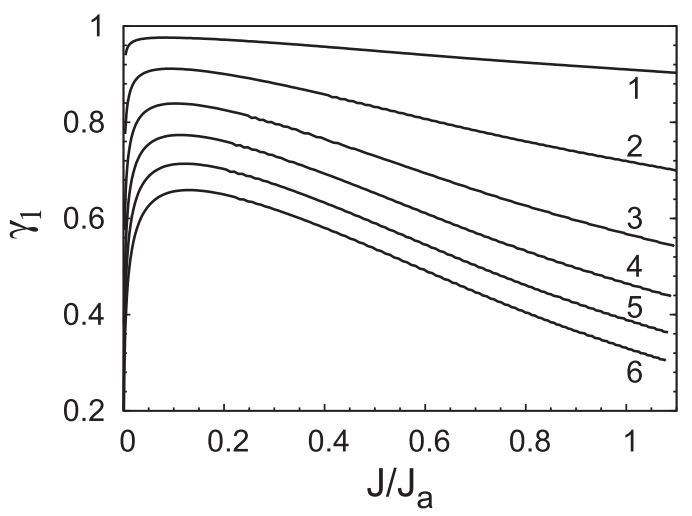

FIG. 5. Radial profiles of $\gamma_{1}$ for runaway electrons: Curve 1 corresponds to $E=1 \mathrm{MeV}$; curve 2 corresponds to $E=5 \mathrm{MeV}$; curve $3, E=10 \mathrm{MeV}$; curve 4, $E=15 \mathrm{MeV}$; curve 5, $E=20 \mathrm{MeV}$, curve $6, E=25 \mathrm{MeV}$. The parameter $\lambda=0.1$. The plasma parameters are the same as in Fig. 2.

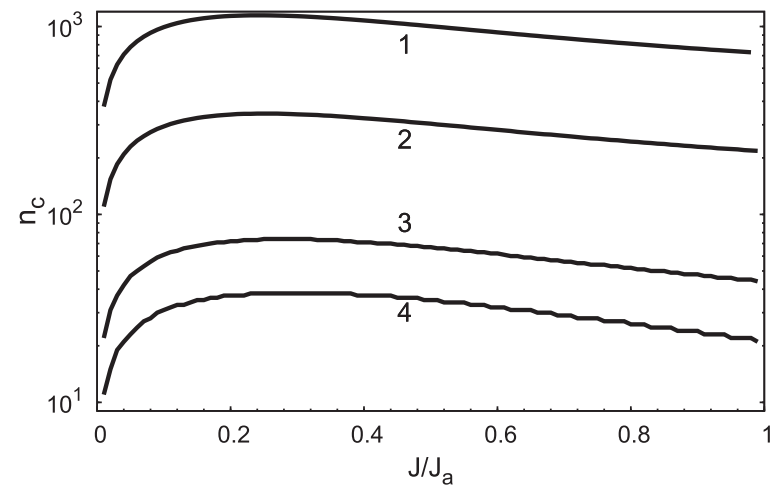

FIG. 6. Radial profiles of $n_{c}$ for runaway electrons: curve 1 corresponds to $E=0.1 \mathrm{MeV}$; curve 2 corresponds to $E=1 \mathrm{MeV}$; curve $3, E=10 \mathrm{MeV}$; curve $4, E=20 \mathrm{MeV}$. The plasma parameters are the same as in Fig. 2.

mainly two kinds of difficulties related to the accuracy and computational times. In the problem of the particle transport in a turbulent field, the number $n$ can be of the order of $10^{2} \div 10^{3}$. It would require to take a very small step $\Delta \varphi$ in the direct numerical integration of the equations of motion for a sufficient accuracy, and therefore, enormous computational times. To avoid these difficulties, we employ the fast mapping method with an integration step equal to one full turn in $\vartheta$ (see Refs. 19 and 50).

Let $P_{k}$ be the $k$-th crossing point of the orbit with the section $\vartheta=0$ as it is schematically shown in Fig. 7. The mapping $P_{k} \rightarrow P_{k+1}$ relates the point $P_{k}$ with the next crossing point $P_{k+1}$. Suppose that $\left(h_{k}, \varphi_{k}, T_{k}\right)$ are the values of the Hamiltonian $H_{0}(J, E)$, the toroidal angle $\varphi$, and the time $T$ at the crossing point $P_{k}$ of the orbit, respectively. We suppose that the fluctuating turbulent field does not change the particle's energy $E$. Then, the simplified form of the mapping $P_{k} \rightarrow P_{k+1}$ reads

$$
\begin{aligned}
h_{k+1} & =h_{k}-\epsilon \frac{\partial P_{k}}{\partial \varphi_{k}} \\
\varphi_{k+1} & =\varphi_{k}+\pi\left[q_{p}\left(h_{k}, E\right)+q_{p}\left(h_{k+1}, E\right)\right]+\epsilon \frac{\partial P_{k}}{\partial h_{k+1}}, \\
T_{k+1} & =T_{k}+\nu_{t}\left(h_{k}, E\right)\left(\varphi_{k+1}-\varphi_{k}\right) / 2 \pi
\end{aligned}
$$

where

$$
P_{k} \equiv P\left(h_{k+1}, E, \varphi_{k}+\pi q_{p}\left(h_{k}, E\right), T_{k}\right)
$$

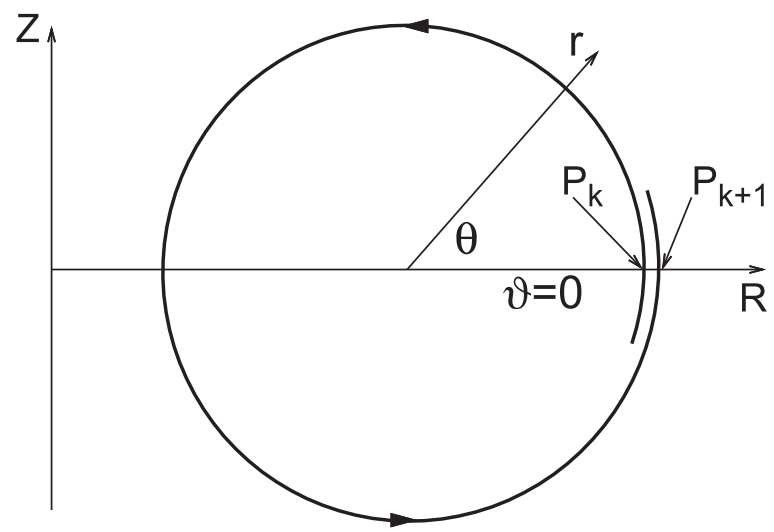

FIG. 7. Schematic view of the mapping, $P_{k} \rightarrow P_{k+1}$. 
is the value of the Poincaré integral $P(h, E, \varphi, T)$, which is taken along the closed contour of the unperturbed orbit:

$$
P(h, E, \varphi, T)=\int_{0}^{2 \pi q_{p}} H_{1}\left(h, E, \vartheta\left(\varphi^{\prime}\right), T\left(\varphi^{\prime}\right), \varphi+\varphi^{\prime}\right) d \varphi^{\prime} .
$$

It can be presented in the form

$$
P(h, E, \varphi, T)=\sum_{n=n_{\min }}^{n_{\max }} R_{n}(h, E) \exp \left(\operatorname{in} \varphi+i \chi_{n}(T)\right),
$$

where $R_{n}(h, E)$ is the Poincaré integral corresponding to the mode number $n$. The latter can be approximated asymptotically with the Fourier coefficients $H_{m n}(J, E)$ and presented as a sum, $R_{n}=R_{n}^{(r e g)}+R_{n}^{(o s c)}$, of the regular $R_{n}^{(r e g)}$ and the oscillatory $R_{n}^{(o s c)}$ parts:

$$
\begin{aligned}
R_{n}^{(r e g)}= & \pi q_{p} H_{n}\left(n q_{p}\right), \\
R_{n}^{(o s c)} & \approx\left(\cos ^{2}\left(\pi n q_{p}\right)-1+i \frac{\sin \left(2 \pi n q_{p}\right)}{2}\right) R_{n}^{(r e g)},
\end{aligned}
$$

where $H_{n}(m) \equiv H_{m n}(J, E)$ is the function defined by extending the value of the integer number $m$ to the continuous one. Note that at the resonant drift surfaces $J_{m n}$, $q_{p}\left(J_{m n}, E\right)=m / n$, the oscillatory part $R_{n}^{(o s c)}=0$. Furthermore, we will omit $E$ in all notations, i.e., $q_{p}(J) \equiv q_{p}(J, E)$.

In order to take into account the finite correlation time $\tau_{c}$, the phases $\chi_{n}$ are randomly changed after $M$ mapping steps, where $M$ is equal to the integer part of the Kubo number $K$, i.e., $M=[K]$.

The optimal number of toroidal modes $n_{\max }$ in Eq. (15) which give the main contribution to the Poincare integral $P(h, E, \varphi)$ is chosen by the following procedure. We calculated the dispersion of the random function $P(h, E, \varphi)$, i.e., $\sigma_{P}=\sqrt{\left\langle P^{2}(h, E, \varphi)\right\rangle}$ as a function of $n_{\max }$. Initially, the dispersion $\sigma_{P}$ grows monotonically with $n_{\max }$ and then reaches its saturation level for $n_{\text {max }}$ exceeding a certain critical number $n_{P}$. The latter depends on the spectrum of the turbulence $c_{n}=n^{-\alpha}$ and on the energy of the electrons. In the calculations, $n_{\text {max }}$ is taken equal to the critical number $n_{P}$.

\section{CHAOTIC TRANSPORT}

A turbulent magnetic field induces the radial transport of the electrons across the drift surfaces. Such a transport is caused by two effects: the fluctuating field with the finite correlation time $\tau_{c}$ and the overlapping of neighboring islands created by the resonance interaction of orbits with $(m, n)$ modes of the turbulent field. The latter case known as chaotic transport appears at amplitudes of the turbulent field exceeding a critical level $\epsilon_{c}$, which depends on the mode content and the turbulent field spectrum $H_{m n}$.

The radial transport of particles is quantified by the diffusion coefficient defined as $D_{r}=\sigma_{0}^{2}(t) / 2 t$, where $\sigma_{0}^{2}(t)$ is the second moment of the radial displacements given by

$$
\sigma_{0}^{2}(t)=\left\langle(r-r(0))^{2}\right\rangle=\frac{1}{N_{t}} \sum_{i=1}^{N_{t}}\left(r_{i}(t)-r_{i}(0)\right)^{2} .
$$

In Eq. (17), $\langle(\cdots)\rangle$ stands for an averaging over the initial phases $\chi_{n}(0), n_{\text {min }} \leq n \leq n_{\max }$, randomly chosen in the interval $[0,2 \pi], r_{i}(t)$ is the radial coordinate of an orbit corresponding to the $i$-th trial, and $N_{t}$ is the total number of trials. All initial coordinates $r_{i}(0)$ are taken on the given drift surface $J=$ constant.

By fitting the dependence of $\sigma_{0}^{2}(t)$ on the time $t$ with the function $2 D_{r} t$ at time intervals $t>t_{K}$ one obtains the diffusion coefficient $D_{r}$, where $t_{K}$ is the decorrelation time of the phases $\varphi$. The latter is determined by the e-folding time of the correlation function of the phases $C_{n}(t)=\langle\exp (\operatorname{in}[\varphi(t)$ $-\varphi(0)])\rangle \sim \exp \left(-t / t_{K}\right)$ at the toroidal mode $n=n_{\text {min }}$. The details of these calculations can be found in Ref. 25 .

\section{A. Quasilinear approximation}

The chaotic diffusion can be described by the quasilinear diffusion coefficient $D(J)$, which can be presented in the following form (see Ref. 19):

$$
D_{r}(J)=v_{\|} R_{0}^{-1}\left(\frac{d r}{d J}\right)^{2} D_{J}, \quad D_{J}=\frac{\epsilon^{2} q_{p}}{8 \pi} \sum_{n=n_{\min }}^{n_{\max }} n^{2}\left|R_{n}^{(r e g)}(J)\right|^{2}
$$

The quantity $D_{J}$ is the quasilinear diffusion coefficient in the action variable $J$, i.e., $D_{J}=\left\langle(J(\varphi)-J(0))^{2}\right\rangle / 2 \varphi$.

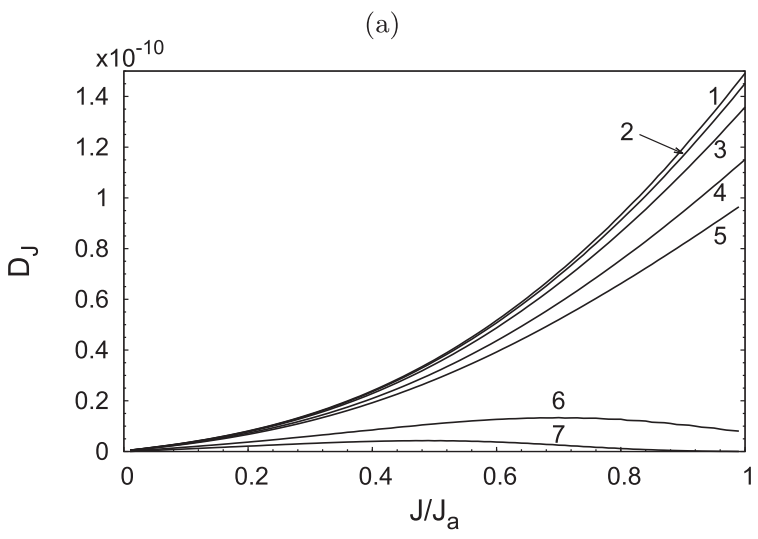

(b)

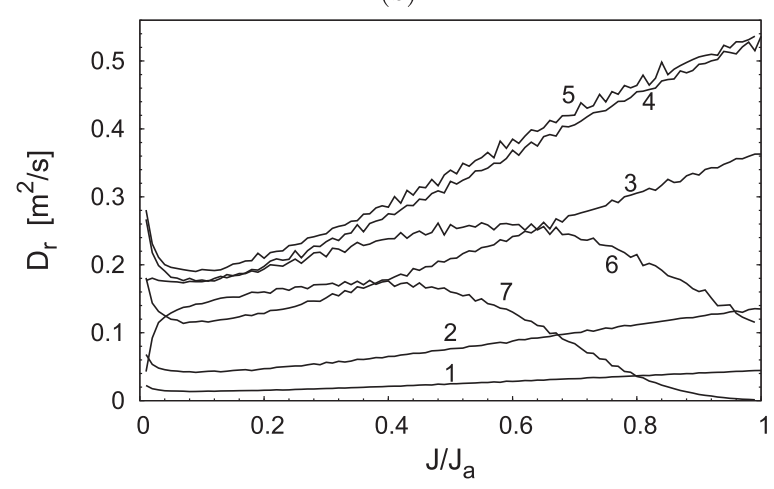

FIG. 8. Quasilinear diffusion coefficients: (a) $D_{J}(J)=\left\langle\left(J-J_{0}\right)^{2}\right\rangle / 2 \varphi$ (18) and (b) $D_{r}(J)=\left\langle(r-r(J))^{2}\right\rangle / 2 t$ for the different runaway electron energies: Curve 1 corresponds to $E=1 \mathrm{keV}$; curve $2, E=10 \mathrm{keV}$; curve 3, $E=10^{2} \mathrm{keV}$; curve $4, E=5 \times 10^{2} \mathrm{keV}$; curve $5, E=1 \mathrm{MeV}$; curve $6, E=10$ $\mathrm{MeV}$; curve 7, $E=20 \mathrm{keV}$. The turbulent level $\epsilon=10^{-4}$, the mode width $w=6$, and the exponent $\alpha=5 / 6$. 
Figures 8(a) and 8(b) show the radial profiles of the quasilinear diffusion coefficients $D_{J}$ and $D_{r}$ in Eq. (18) for the different electron energies. The intervals of the modes are chosen for $n_{\min }=10 \leq n \leq n_{\max }=n_{c}(\mathrm{~J}) \sim 10^{2}$, where $n_{c}(J)$ is plotted in Fig. 6 (curve 2). The exponent $\alpha$ is taken equal to $5 / 6$, which corresponds to the power spectrum $\left|H_{m n}\right|^{2} \sim n^{-5 / 3}$ and the turbulence level $\epsilon=10^{-4}$, which corresponds to the magnetic turbulence level $\delta B$ shown by curve 1 in Fig. 4.

As seen from Fig. 8(a), the diffusion coefficient $D_{J}$ characterizing the chaotic transport of the orbits takes the highest values for electrons with low energies. At the electron energies between $E=1 \mathrm{keV}$ and $E=10^{2} \mathrm{keV}$ (curves $1-3$ ), $D_{J}$ practically coincides with the diffusion coefficients of the field lines. By increasing the electron energies, the diffusion coefficients $D_{J}$ monotonically decrease as shown by the curves 4-7 corresponding to electron energies from $500 \mathrm{keV}$ to $20 \mathrm{MeV}$. This is due to a weakening the effect of the magnetic turbulence on the runaway electrons by increasing their energy due to the growth of the deviations of the orbits from the magnetic surfaces and the gyro-averaging.

The diffusion coefficients $D_{r}$ characterizing the transport rate along the radial coordinate $r$ change non-monotonically with the energy. Since $D_{r} \sim v_{\|} D_{J}$, the diffusion coefficient $D_{r}$ grows in the energy interval from $1 \mathrm{keV}$ to $1 \mathrm{MeV}$ because of the increase of the parallel velocity $v_{\|}$with the energy $E$ and reaches the maximal value at $E \sim 1 \mathrm{MeV}$. If the energy is increased further $D_{r}$ starts to decrease due to the decay of $D_{J}$ while $v_{\|}$approaches the speed of light $c$.

\section{B. Numerical diffusion coefficients}

Now, we consider the radial profiles of the numerically calculated diffusion coefficients $D_{r}$. The dependence of $D_{r}$ on the action variable $J$ is presented in Fig. 9 for the runaway electrons of the energy $E=1 \mathrm{MeV}$ at the turbulent field parameters as in Fig. 8: curve 1 corresponds to the quasilinear prediction (18), curve 2 describes the numerically calculated $D_{r}$, and curve 3 [right-hand axis] corresponds the radial

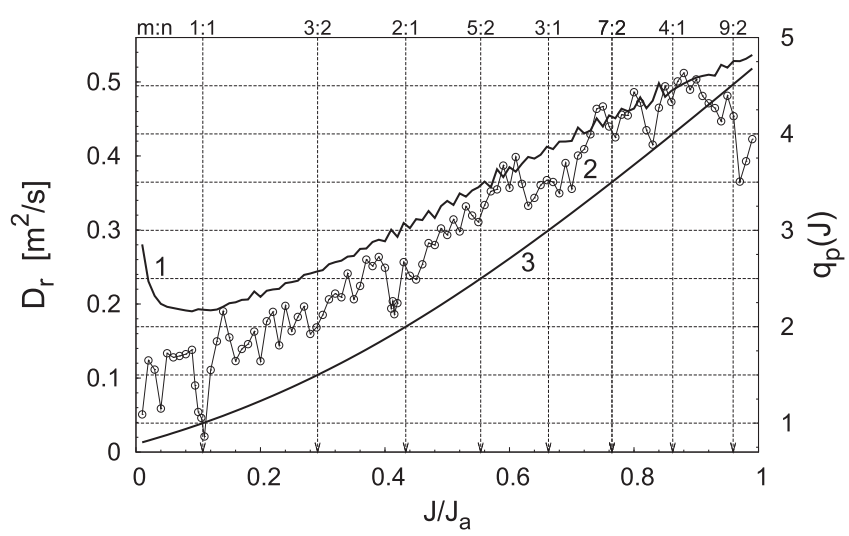

FIG. 9. Radial profile of diffusion coefficient $D_{J}$ for the runaway electron energy $E=1 \mathrm{MeV}$. Curve 1 corresponds to the quasilinear prediction, curve 2 corresponds to the mapping results with the Poincaré functions $R_{n c}=\pi q_{p} K_{n q_{p}, n}, R_{n s}=0$; curve 3 describes the effective safety factor profiles, $q_{p}(J)$ [right-hand axis]. The positions of the low order resonant drift surfaces, $J_{m n}$, are shown by vertical straight lines. The number of orbits $N=10^{3}$, the toroidal modes $10 \leq n \leq n_{c}, J_{a}=3.32658 \times 10^{-2}$. profile of the effective safety factor $q_{p}(J)$. The vertical and horizontal lines describe the positions of the low-order rational drift surfaces $J_{m n}, q_{p}\left(J_{m n}\right)=m / n$. The corresponding radial profile of the Kubo number was shown in Fig. 3 (curve 2). It is of order of $K \sim 100$. At the chosen turbulence level $\epsilon$ and the large Kubo number $K$ of the order of $K \sim 10^{2}$, the radial transport is mainly caused by the chaotic diffusion due to the overlapping of neighboring resonances.

As seen from Fig. 9, the radial profile of $D_{r}$ (curve 2) has a fractal-like dependence which in average follows the quasilinear prediction (18). One can see that near the loworder rational drift surfaces (vertical lines), $m / n=1,3 / 2,2 / 1$, $5 / 2$, and $3 / 1$, the diffusion coefficient drops to lower values significantly deviating from the quasilinear values. One can say that effective barriers to the radial transport are formed. At the given turbulence level $\epsilon$, the width and depth of these barriers depend on the exponent $\alpha$ in the power-law distribution of the toroidal modes $c_{n}=n^{-\alpha}$. For example, at $\alpha=5 / 6$ the barrier near the rational surface $m: n=2: 1$ is rather small (see Fig. 9). For a larger value of $\alpha$ when the contribution of higher toroidal modes $n$ is decreased the transport barrier becomes wider and deeper. It is shown in Fig. 10 where the radial profiles of $D_{r}$ are plotted for two different values of $\alpha$ the rational drift surface $m: n=2: 1$. Curve 1 corresponds to $\alpha=4 / 3$ for the turbulence level $\epsilon=10^{-4}\left(D_{r}\right.$ is multiplied by the factor 10), curve 2 corresponds to the same $\alpha=4 / 3$ but $\epsilon=\sqrt{10} \times 10^{-4}$, and curve 3 corresponds to the same $\alpha=1$ for $\epsilon=10^{1 / 6} \times 10^{-4}$.

The reduced radial transport near the low-order rational drift surfaces is related to the gaps in their density there. As shown in Appendix, the gap width $\Delta w$ is inversely proportional to the maximal toroidal mode number $n_{\max } \sim n_{c}$ for which the corresponding terms $R_{n}$ in the function (15) are not negligible. One would expect that with increasing the energy of the electrons the transport barriers become wider and deeper since the number of the toroidal modes $n>n_{c}$ contributing to the transport effectively decays. This is shown in Figs. 11(a) and 11(b) where the radial profiles of

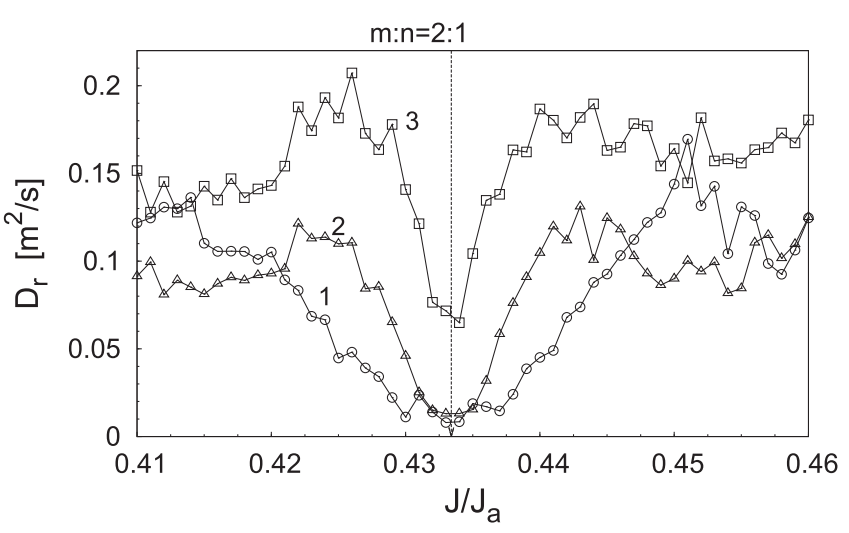

FIG. 10. Radial profile of the diffusion coefficient $D_{r}$ for the runaway electron of energy $E=1 \mathrm{MeV}$ near the rational drift surface $q_{p}=2$ for the several values of the exponent $\alpha$ in the power-law $c_{n}$ : curve $1\left(10 \times D_{r}\right)$ corresponds to $\alpha=4 / 3$ at the perturbation level $\epsilon=10^{-4}$, curve 2 corresponds to $\alpha=4 / 3$ at the perturbation level $\epsilon=\sqrt{10} \times 10^{-4}$, curve 3 corresponds to $\alpha=1$ at the perturbation level $\epsilon=10^{1 / 6} \times 10^{-4}$. The position of the rational drift surface $q_{p}=2, J_{m n}$, is shown by vertical straight lines. 
(a)

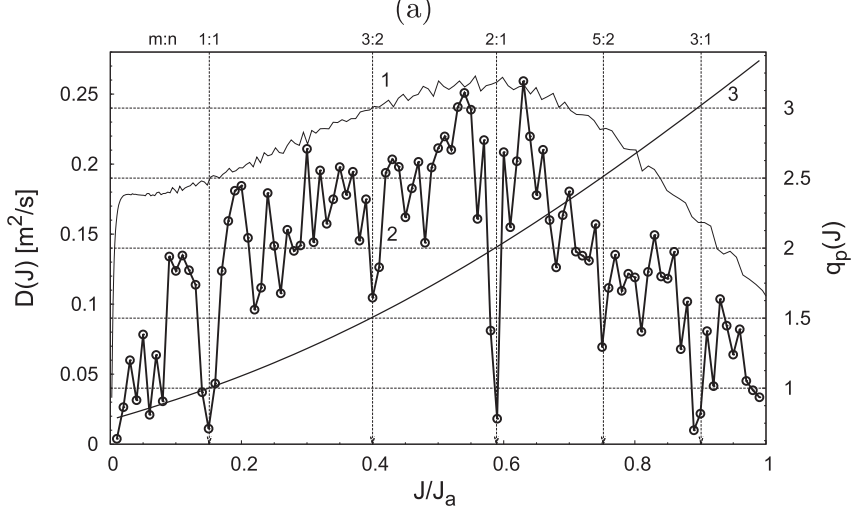

(b)

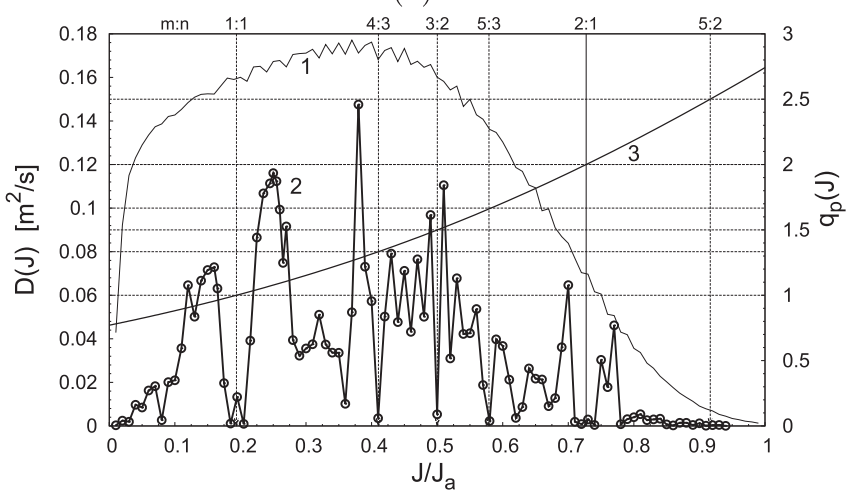

FIG. 11. The same as in Fig. 9 but for the electron energies $E=10 \mathrm{MeV}$ (a) and $E=20 \mathrm{MeV}$ (b). The other parameters are the same as in Fig. 9.

the diffusion coefficients for the runaway energies $E=10$ $\mathrm{MeV}$ and $E=20 \mathrm{MeV}$ are plotted.

As seen from Figs. 11(a) and 11(b), there are also regions outside the low-order rational surfaces where the diffusion coefficients are much smaller than the quasilinear values. In these regions, the radial transport is caused mainly by the fluctuations of the turbulent field in time. There, the chaotic transport is reduced or absent because of the low density of the rational surfaces (not gaps) which is not sufficient for an overlapping of the resonances. However, when one approaches the low-order rational surfaces the diffusion coefficients grow to their local maximal values and then drop drastically. The radial profile of $D_{r}$ near these areas is similar to the behavior of the density $\rho(q, N)$ near low-order rational numbers $q=m / n$ as it is shown in Fig. 13.

\section{Effect of isolated MHD modes}

MHD modes are frequently excited in real tokamak discharges. Typically, the MHD modes with low poloidal and toroidal mode numbers $(m, n)$ are isolated and do not disrupt the plasma. They are localized near the rational magnetic surfaces $J_{m n}, q\left(J_{m n}\right)=m / n$. These MHD modes may significantly influence the chaotic transport caused by the microturbulence field near the corresponding rational drift surfaces.

In the presence of the $(m, n)$ MHD mode alone, the structures of the field lines and particle orbits near the corresponding rational surfaces are modified. Particularly, it creates an island which traps the particles with the appropriate initial conditions. The particle motion can be characterized by introducing the averaged safety factor defined by

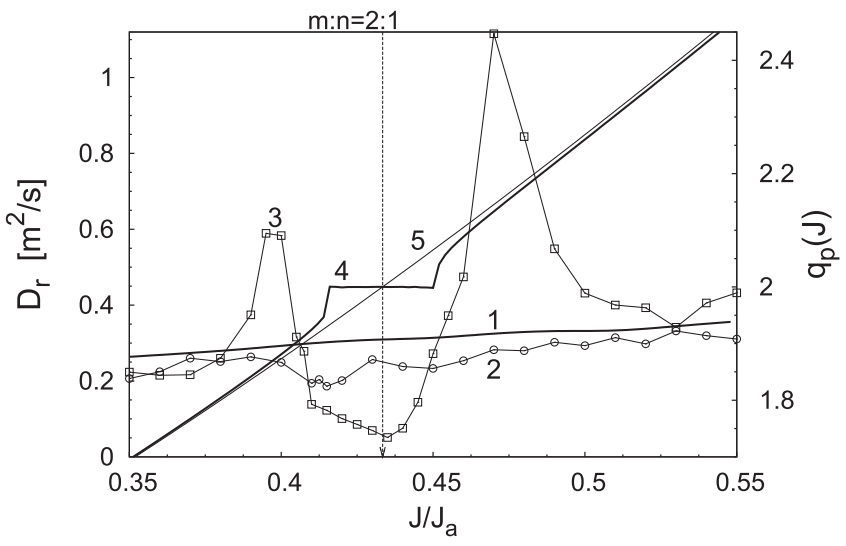

FIG. 12. The same as in Fig. 9 but in the presence of the isolated $(m, n)=(2: 1)$ MHD mode: Curve 1 corresponds to the quasilinear prediction, curves 2 and 3 correspond to the numerical results without and with MHD mode, respectively. Curves 4 and 5 [right-hand axis] describe the averaged, $q_{a v}(J)$, and effective, $q_{p}(J)$, safety factors, respectively. The amplitude of the MHD mode is taken equal to $\epsilon=10^{-4}$. The other parameters are the same as in Fig. 9.

$$
q_{a v}=\frac{1}{N} \sum_{k=0}^{N} \frac{\varphi_{k+1}-\varphi_{k}}{2 \pi}, \quad N \gg 1
$$

where $\varphi_{k}(k=0,1,2, \ldots, N)$ is the sequence of the toroidal angles in the mapping (12) and the averaging is done over large number of mapping iterations $N, N \gg 1$. The radial profile of $q_{a v}(J)$ is flattened in the region $J_{m n}$ $-W_{m n} / 2<J<J_{m n}+W_{m n} / 2$ near the resonant surface $J_{m n}$, where $W_{m n}=4\left[\epsilon H_{m n} / d q_{p}^{-1} / d J\right]^{1 / 2}$ is the island width. According to (A1) and (A2), such a profile of $q_{a v}$ leads to a widening of the gap in the density of the rational surfaces, and thus reduces the chaotic transport due to interactions with the modes of the turbulent field.

Figure 12 shows the example of a reduced turbulent transport near the $(m: n)=(2: 1)$ rational surface in the presence of the corresponding MHD mode: Curve 1 describes the quasilinear diffusion coefficients $D_{r}$, curves 2 and 3 correspond to the numerical $D_{r}$ without and with MHD mode, respectively. Curves 4 and 5 [right-hand axis] describe the

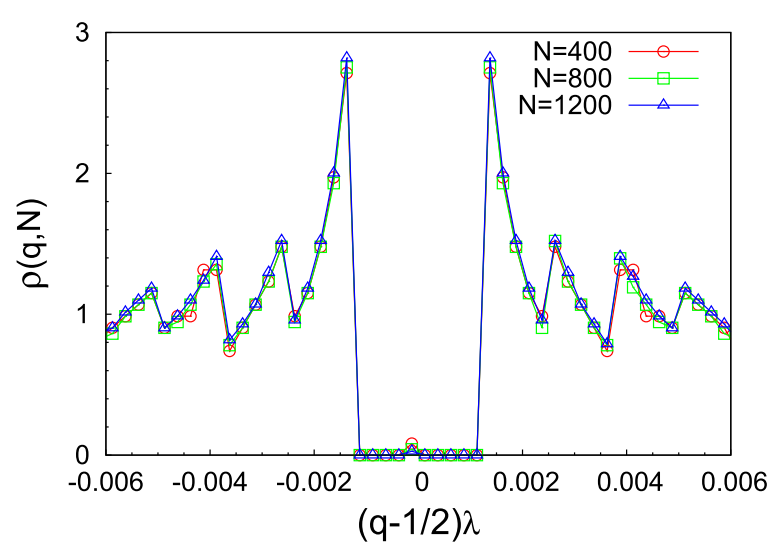

FIG. 13. Density of rational numbers near $q=1 / 2$ for the different $N$ at the fixed $M=1$. It also shows the rescaling law (A2) of the distribution density $\rho(q, M, N)$ for the different numbers $N$ near the $q=1 / 2$. The rescaling parameters $\lambda=400 / N$. 
averaged, $q_{a v}(J)$, and the effective, $q_{p}(J)$, safety factors, respectively. It is assumed that the MHD mode has a localized radial structure given by Eq. (7) and the amplitude of the MHD mode is taken equal to $\epsilon=10^{-4}$. The electron energy $E$ is taken as $1 \mathrm{MeV}$.

As seen from Fig. 12, there is a strong reduction of the turbulent diffusion near the rational surface where $q_{a v}$ is flattened (see curve 4). The width of the transport barrier is of the order of the width of the corresponding island $W_{m n}$. However, the diffusion has large spikes just outside the island. It is reduced to the quasilinear level (curve 1) in the regions far from the island.

\section{CONCLUSION}

In conclusion, we have studied the radial transport of runaway electrons in a toroidal system in the presence of a background turbulent magnetic field. It is assumed that such a turbulent field has a wide spectrum of modes with a finite correlation time. The numerical calculations of diffusion coefficients are carried out using the fast symplectic mapping constructed from the Hamiltonian equations for the relativistic guiding-center motion of the electrons. The latter takes both effects into account, the deviation of the electron drift orbits from the magnetic surfaces and the gyro-averaging of the turbulent magnetic field.

Since the runaway electrons have large Kubo numbers, their radial transport in a turbulent magnetic field is mainly caused by the chaotic transport due to overlapping islands, which are formed by the interactions of particle orbits with the resonant components of the turbulent field. The numerical calculations show that the radial profiles of the diffusion coefficients of the chaotic transport have fractal-like structures with a reduced diffusivity near the low-order rational drift surfaces thus forming transport barriers for the radial transport. The fractal-like dependence of the runaway electron diffusion stems from the large values of the corresponding Kubo number when the high speed electrons "feel" the structure of the turbulent magnetic field. The width and depth of the transport barriers depend on the toroidal mode spectrum $c_{n}=n^{-\alpha}$ as well as on the energy of the runaway electrons. The barriers become more pronounced by increasing the electron energy. The latter is caused not only by the cutting out of higher modes by gyro-averaging but also by the increase of the Kubo number.

The described mechanism of the turbulent transport of energetic electron can be one of the main reasons for the improved confinement of runaway electrons in tokamaks additional to the effects of the gyro-averaging and the orbitaveraging mechanisms. One can expect that this effect may lead to the formation of the nested beams of runaway electrons. To study such a possibility, one should study processes of the generation and acceleration of electron beams in the presence of magnetic micro-turbulence perturbations.

\section{ACKNOWLEDGMENTS}

Authors are grateful to M. Tokar and D. Reiser for stimulating discussions and comments.

\section{APPENDIX: DENSITY OF RATIONAL DRIFT SURFACES}

Here, we briefly describe the density of the rational surfaces and its property. The detailed description of this problem is given in Ref. 25 .

Let $J_{m n}$ be resonant values of the drift surfaces, $q\left(J_{m n}\right)=m / n$, corresponding to the mode numbers $(m, n)$. Assume that the interval of the toroidal modes is $M \leq n \leq N$. Let $\Delta P$ be a number rational surfaces $J_{m n}$ in the interval $J, J+\Delta J$. Then, the density $\rho(J)$ of rational surfaces is defined as $\rho(J, M, N)=\Delta P / \Delta J$. One can also introduce the density of the rational numbers $q=m / n, \rho(q, M, N)$, as follow $\rho(J, M, N)=\Delta P / \Delta J$. The relation between $\rho(J, M, N)$ and $\rho(q, M, N)$ is given by

$$
\rho(J, M, N)=\rho(q, M, N) \frac{d q}{d J} .
$$

We have numerically calculated the density function $\rho(q, M, N)$ in the interval $0 \leq q \leq 1$ for different values of $N$. It shows that the distribution density function has indeed gaps near the low-order rational numbers $q_{m n}=m / n: 0 / 1,1 / 1,1 / 2$, $1 / 3$, and $2 / 3$. Moreover, $\rho(q, M, N)$ has a self-similarity behavior near these numbers $q_{m n}$. The self-similarity of $\rho(q, N) \equiv \rho(q, 1, N)$ is expressed by the following rescaling law:

$$
\rho\left(q-q_{m, n}, N\right) \approx \rho\left(\frac{q-q_{m, n}}{\lambda}, \lambda N\right),
$$

where $\lambda$ is a constant. The gaps and the rescaling law for the distribution density $\rho(q, N)$ near $q=1 / 2$ are illustrated in Fig. 13. From the rescaling property (A2), it follows that the gap width $\Delta w$ is inversely proportional to the number $N$.

${ }^{1}$ H. E. Mynick and J. Strachan, Phys. Fluids 24, 695 (1981).

${ }^{2}$ J. R. Myra, P. J. Catto, A. J. Wootton, R. D. Bengtson, and P. W. Wang, Phys. Fluids B 4, 2092 (1992).

${ }^{3}$ J. R. Myra, P. J. Catto, H. E. Mynick, and R. E. Duvall, Phys. Fluids B 5, 1160 (1993).

${ }^{4}$ M. de Rover, N. J. Lopes Cardozo, and A. Montvai, Phys. Plasmas 3, 4468 (1996).

${ }^{5}$ M. de Rover, N. J. Lopes Cardozo, and A. Montvai, Phys. Plasmas 3, 4478 (1996).

${ }^{6}$ M. de Rover, A. M. Schilham, A. Montvai, and N. J. Lopes Cardozo, Phys. Plasmas 6, 2443 (1999).

${ }^{7}$ J. R. Martín-Solís, R. Sánchez, and B. Esposito, Phys. Plasmas 6, 3925 (1999).

${ }^{8}$ T. Hauff and F. Jenko, Phys. Plasmas 16, 102308 (2009).

${ }^{9}$ R. D. Gill, Nucl. Fusion 33, 1613 (1993).

${ }^{10}$ R. Jaspers et al., Nucl. Fusion 36, 367 (1996).

${ }^{11}$ P. Helander, L.-G. Eriksson, and F. Andersson, Plasma Phys. Controlled Fusion 44, B247 (2002).

${ }^{12}$ L.-G. Eriksson, P. Helander, F. Andersson, D. Anderson, and M. Lisak, Phys. Rev. Lett. 92, 205004 (2004).

${ }^{13}$ V. V. Plyusnin et al., Nucl. Fusion 46, 277 (2006).

${ }^{14}$ M. Lehnen et al., J. Nucl. Mater. 390-391, 740 (2009).

${ }^{15}$ O. Kwon et al., Nucl. Fusion 28, 1931 (1988).

${ }^{16}$ I. Entrop, N. J. Lopes Cardozo, R. Jaspers, and K. H. Finken, Phys. Rev. Lett. 84, 3606 (2000).

${ }^{17}$ A. B. Rechester and M. N. Rosenbluth, Phys. Rev. Lett. 40, 38 (1978).

${ }^{18}$ J. R. Myra and P. Catto, Phys. Fluids B 4, 176 (1992).

${ }^{19}$ S. S. Abdullaev, K. Finken, T. Kudyakov, and M. Lehnen, Contrib. Plasma Phys. 50, 929 (2010).

${ }^{20}$ R. Yoshino and S. Tokuda, Nucl. Fusion 40, 1293 (2000).

${ }^{21}$ B. Esposito et al., Plasma Phys. Controlled Fusion 38, 2035 (1996).

${ }^{22}$ P. Helander, L.-G. Eriksson, and F. Andersson, Phys. Plasmas 7, 4106 (2000). 
${ }^{23}$ R. Sánchez, J. R. Martín-Solís, and B. Esposito, Comput. Phys. Commun. 156, 95 (2003).

${ }^{24}$ O. Bakunin, Turbulence and Diffusion: Scaling Versus Equations (Springer, Berlin/Heidelberg, 2008).

${ }^{25}$ S. S. Abdullaev, Phys. Rev. E 84, 026204 (2011).

${ }^{26}$ A. Beklemishev and W. Horton, Phys. Fluids B 4, 200 (1992).

${ }^{27}$ C. C. Hegna and J. D. Callen, Phys. Fluids 5, 1804 (1993).

${ }^{28}$ N. J. L. Cardozo et al., Plasma Phys. Controlled Fusion 39, B303 (1997).

${ }^{29}$ G. Hogeweij, N. L. Cardozo, M. D. Baar, and A. Schilham, Nucl. Fusion 38, 1881 (1998).

${ }^{30} \mathrm{R}$. Brakel and W7-AS Team, Nucl. Fusion 42, 903 (2002).

${ }^{31}$ P. Gohil et al., Nucl. Fusion 43, 708 (2003).

${ }^{32}$ L.-G. Eriksson et al., Phys. Rev. Lett. 88, 145001 (2002).

${ }^{33}$ X. Garbet et al., Nucl. Fusion 43, 975 (2003).

${ }^{34}$ K. A. Razumova et al., Plasma Phys. Controlled Fusion 45, 1247 (2003).

${ }^{35}$ N. L. Cardozo, Trans. Fusion Sci. Technol. 45, 321 (2004).

${ }^{36} \mathrm{G}$. Hogeweij et al., Nucl. Fusion 44, 533 (2004).

${ }^{37}$ E. Joffrin et al., Nucl. Fusion 43, 1167 (2003).

${ }^{38}$ M. E. Austin et al., Phys. Plasmas 13, 082502 (2006).

${ }^{39}$ K. Razumova et al., Plasma Phys. Controlled Fusion 50, 105004 (2008).
${ }^{40}$ S. S. Abdullaev and K. H. Finken, Phys. Plasmas 9, 4193 (2002).

${ }^{41}$ S. S. Abdullaev, A. Wingen, and K. H. Spatschek, Phys. Plasmas 13, 042509 (2006)

${ }^{42}$ A. Wingen, S. S. Abdullaev, K. H. Finken, and K. H. Spatschek, Nucl. Fusion 46, 941 (2006).

${ }^{43}$ K. H. Finken et al., Nucl. Fusion 30, 859 (1990).

${ }^{44}$ R. J. E. Jaspers, "Relativistic runaway electrons in tokamak plasmas," $\mathrm{Ph} . \mathrm{D}$. thesis (Eidenhoven University of Technology, The Netherlands, 1995).

${ }^{45}$ J. F. Drake, N. T. Gladd, C. S. Liu, and C. L. Chang, Phys. Rev. Lett. 44, 994 (1980).

${ }^{46}$ H. Doerk, F. Jenko, M. J. Pueschel, and D. R. Hatch, Phys. Rev. Lett. 106, 155003 (2011)

${ }^{47}$ M. Vlad and F. Spineanu, Plasma Phys. Controlled Fusion 47, 281 (2005).

${ }^{48}$ M. Vlad, F. Spineanu, S.-I. Itoh, M. Yagi, and K. Itoh, Plasma Phys. Controlled Fusion 47, 1015 (2005).

${ }^{49}$ P. Pommois, P. Veltri, and G. Zimbardo, Phys. Rev. E 63, 066405 (2001).

${ }^{50}$ S. S. Abdullaev, Construction of Mappings for Hamiltonian Systems and Their Applications (Springer, Berlin/Heidelberg, 2006). 